\title{
A Novel OCT Design for Cultural Heritage Applications
}

Danielle Duggins ${ }^{1}$, Fengqiang $\mathrm{Li}^{2}$, Maurice Aalders ${ }^{3}$, Oliver Cossairt ${ }^{2}$, Aggelos Katsaggelos ${ }^{2}$, and Marc Walton $^{4}$

1. Northwestern University, Department of Materials Science \& Engineering, Evanston, IL, USA

2. Northwestern University, Department of Electrical Engineering and Computer Science, Evanston, IL, USA

3. University of Amsterdam, Department of Biomedical Engineering and Physics, Academic Medical Center, Amsterdam, Netherlands

4. Northwestern University/Art Institute of Chicago Center for Scientific Studies of the Arts (NUACCESS), Evanston, IL, Chicago, USA

The object challenges presented by cultural heritage materials can serve as catalysts for the development of novel characterization techniques. Generally, these objects are delicate, often with insufficient historical documentation, and are characterized by complex heterogeneous material systems. Because these objects require non-destructive and non-contact characterization methods, most analyses are limited to surface investigations only, rendering information about structural and geometric properties unobtainable. Further constraints on characterization techniques are imposed by restricted exposure to light to prevent degradation of the object surface. Additionally, many objects must be analyzed in situ, producing further complications for instrumentation design.

These challenges are not unique to cultural heritage; the need for non-invasive analyses of delicate samples, for example, is a well-known criterion in biomedical research. Indeed, there is the potential for a rich exchange of instrumentation and methodology between cultural heritage and the biomedical sciences. One such adaptation of a biomedical technique, optical coherence tomography (OCT), which was developed nearly thirty years ago and is virtually ubiquitous in ophthalmology today [1], has been successfully applied to cultural heritage investigations [2-4].

Optical coherence tomography is a low-coherence broadband interferometric technique that provides structural information of materials at resolutions less than $20 \mu \mathrm{m}$. The reflected light coming from interfaces within the sample produce an interference signal only when the distance traveled in the reference arm and sample arms is of similar length. In ophthalmology, it is used to image the retina and is often referred to as the optical equivalent of ultrasound. This is a useful analogy for understanding the technique: the data it produces are virtual cross-sections of structural information. OCT is flexible and well-suited for a range of sample environments. OCT designs utilize sources ranging from visible light to near-infrared wavelengths and are able to image samples in situ and through turbid media. Most importantly, OCT is a non-invasive, non-contact, and non-destructive technique; requires little time per scan; and uses very low levels of light, making it suitable for analyses of cultural objects.

This work presents a lab-built OCT system specifically designed for the analysis of paintings. A key consideration in adapting this technique is the need for a suitable wavelength range in which paint media, rather than biological tissues, are transparent, while good imaging depth and axial resolution are maintained. Additionally, while high acquisition speed is paramount for subjects in vivo, museum objects can be analyzed over reasonably long periods. 
Following the system developed by Cheung et al. [5], the center wavelength of our system, $2 \mu \mathrm{m}$, is longer than typical OCT systems. Most paint pigments have low absorption properties in this range [6], allowing the light to penetrate deeper into the sample and take advantage of the full depth range of the system. However, the depth resolution is proportional to the square of the center wavelength, so increasing the center wavelength decreases the depth resolution of the system [7]. We compensate for the longer center wavelength and recover a reasonable depth resolution by maintaining a large bandwidth in the system $(400 \mathrm{~nm})$.

In this spectral-domain OCT, the spectrometer specifications define the imaging depth and the scan speed of the system. Spectrometers in this wavelength range can be expensive and have a limited number of pixels, which would limit the imaging depth of the system. To address this issue, we incorporate a spectrometer containing a digital micromirror device (DMD) (Texas Instruments), which can scan over the entire wavelength range and only requires a single detector. This allows us to obtain a large number of pixels while keeping costs low.

Through the use of mostly reflective optics and a supercontinuum laser source (NKT Photonics), we can maintain a large bandwidth, and a high resolution. The theoretical axial resolution of this system is 8.7 $\mu \mathrm{m}$ in air. By incorporating the DMD-based spectrometer we can obtain a theoretical imaging depth of $1.1 \mathrm{~mm}$. The novel adaptations we have designed in this system make it ideal for obtaining virtual crosssections of a painting's structure.

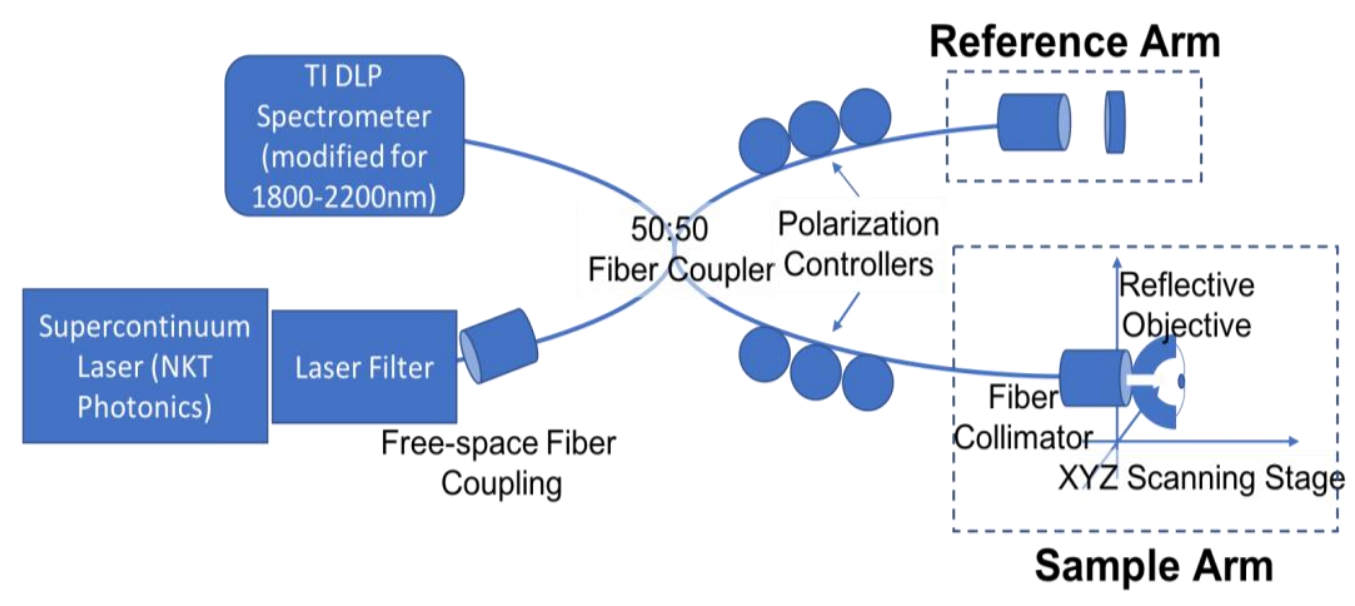

Figure 1. Simplified Design of OCT Setup.

References:

[1] D Huang et al, Science 254 (1991) p. 1178-1181.

[2] M Yang et al, Archaeometry 46 (2004) p. 171-182.

[3] P Targowski et al, Stud. Conserv. 49 (2004) p. 107-114.

[4] H Liang et al, Second European Workshop on Optical Fibre Sensors 5502, 378-382 (International Society for Optics and Photonics, 2004).

[5] C Cheung et al, Optical Express 23 (2015) p. 1992.

[6] H Liang et al, Appl. Phys. B 111 (2013), p. 589-602.

[7] P Targowski and M Iwanicka Appl. Phys. A: Mater. Sci. Process. 106 (2012), p. 265-277.

[8] The authors gratefully acknowledge support from the Andrew W. Mellon Foundation and the National Science Foundation, Office of International Science \& Engineering (Award \#1743748). 